\title{
Der Point-of-Care-Tarif: eine Übersicht
}

Anton Prantl

Direktionspräsident Ärztekasse

Korrespondenz:

Anton Prantl

Ärztekasse

Steinackerstrasse 35

CH-8902 Urdorf

geschaeftsleitung@aerztekasse.ch

\section{Zusammenfassung}

Das Bundesamt für Gesundheit (BAG) führt in seinem Bericht zur Vernehmlassung der Analysenliste als Motivation für deren Revision den massiven technologischen Fortschritt seit 1994 und sich bereits abzeichnende künftige Änderungen wie Internationalisierung, technischen Fortschritt und Beschaffungsverhalten der Grosslaboratorien an.

Weiter stipuliert das BAG, einerseits hätten die Preise der Untersuchungen unabhängig vom Erbringungsort gleich zu sein und andererseits verordnet es aber im gleichen Atemzug Grosslabors eine Grundtaxe von Fr. 24.- pro Laborauftrag und den Praxislabors eine Präsenztaxe von Fr. 8.-. Diese betriebswirtschaftliche Inkonsequenz persifliert umgehend das gesamte Tarifwerk: Wird die am häufigsten im Praxislabor erbrachte Einzelanalyse nicht mehr dort erbracht, sondern ans Grosslabor abgegeben, kostet diese Untersuchung den Versicherten nicht mehr wie bisher Fr. 12.60, sondern eben dank der Grundtaxe Fr. 29.- neben dem ökologischen Unsinn, Proben durch die ganze Schweiz zu «chauffieren», ein happiger Kostenschub von 230\%.

Die so errechneten Vergütungen (inklusive Präsenztaxe) führen zu Preissenkungen um 30\% für das typische Praxislabor nicht verkraftbar.

Als Konsequenz war damit die Notwendigkeit gegeben, eine auf die Eckwerte der Präsenzdiagnostik aufbauende Kalkulation zu entwickeln. Dies ist aufgrund der wie immer ausgezeichneten Datenlage bei den freipraktizierenden Ärzten keine Hexerei; die Integration der damit für die Präsenzdiagnostik speziell tarifierten Analysen in die Analyseliste ist kein Problem, denn diese kennt bereits heute mehrere solche Segmentierungen.

Wie die Kontrollrechnung zeigt, halten sich die Auswirkungen des Point-of-Care-Tarifes (POCTarifes) in engen Grenzen - gegenüber dem heutigen Tarif weist die Kontrollrechnung eine Differenz von rund 3\% aus: ein zufälliges, aber glückliches Resultat, beachtet man die durch die betriebswirtschaftliche Berechnungsmethodik doch erhebliche Preisdifferenzierung zwischen den Analysekategorien.

\section{Ausgangslage}

Mehrere Unstimmigkeiten im Zusammenhang mit der Revision der Analyseliste haben die FMH veranlasst, die Situation Praxislabor aufzuarbeiten:

\section{Revisionsgrundlage}

Das BAG führt in seinem Bericht zur Vernehmlassung der Analysenliste als Motivation der Revision den massiven technologischen Fortschritt seit 1994 und sich bereits abzeichnende künftige Änderungen wie Internationalisierung, technischer Fortschritt und Beschaffungsverhalten der Grosslaboratorien an.

Diese Sachverhalte mögen für Grosslaboratorien zutreffen, die auf Massenproduktion ausgerichtet sind. Im typischen Praxislabor sind Rationalisierungsgewinne aus der Natur der Sache nicht realisierbar. Weder bei den Geräten noch bei den Reagenzien sind für das Praxislabor tiefgreifende Veränderungen auszumachen.

\section{Eine Untersuchung - ein Preis}

Das BAG hält beharrlich daran fest, dass es für Analysen nur eine Berechnungsmethode bzw. einen Preis geben könne. Genau dieser selbst konstruierten Forderung kommt das BAG jedoch nicht nach, indem es neben der Analyse auch eine erbringerspezifische Grundtaxe tarifiert. Für den Versicherten entstehen somit abhängig vom Ort der Leistungserbringung unterschiedliche Preise; typischerweise höhere, wenn das, was heute im Praxislabor ausgeführt wird, neu im Grosslabor erbracht werden muss.

Ein Rechenbeispiel an einer der häufigsten im Praxislabor erbrachten Leistungen macht dies deutlich: Heute kostet ein CRP im Praxislabor Fr. 10.80. Neu wird die Analyse gemäss den Berechnungen des BAG wohl nur noch Fr. 9.kosten. Wird sie aber im Grosslabor ausgeführt, addieren sich Fr. 24.- Grundtaxe dazu!

\section{Massive Differenzen in den Kalkulationsgrundlagen}

Gemäss unserem momentanen Kenntnisstand rechnet das BAG die für das Praxislabor relevanten Analysen mit einem für das Grosslabor entwickelten Modell und behauptet, mit einer pro Laborsitzung gewährten Präsenztaxe die Eigenheiten des Praxislabors betriebswirtschaftlich korrekt abzubilden. Daraus resultiert gegenüber heute eine 20-30\% tiefere Vergütung für das Praxislabor. Pikanterweise liegt dieselbe Taxe für das Grosslabor gut dreimal höher!

Zur Festlegung obiger Pauschale bleiben die Aussagen des BAG recht vage; angeblich leitet das BAG diese aus den Kalkulationen der Workflowanalyse ab. Deren Autoren haben sich unzweideutig (auch gegenüber dem BAG) dahin- 
gehend geäussert, dass ihre Berechnungen einen Deckungsbeitrag und keine Vollkostenrechnung darstellen, also vom BAG unzutreffend verwendet werden.

\section{Eine Zuschlagstaxe ist betriebswirtschaftlich nicht haltbar}

Ein pauschaler Zuschlag pro Sitzung ist nur dann akzeptabel, wenn er im Verhältnis zur produktiven Leistung klein, auf einen homogenen Mix angewendet wird und nur stufengerechte Elemente vergütet. All dies trifft auf die vorgesehene Präsenztaxe nicht zu:

- Die Präsenztaxe übersteigt die eigentliche Leistung im Falle des Urinstatus, der Leistungen der klinischen Chemie und der Blutsenkung teils um gut das Doppelte.

- Analysemix und Anzahl Analysen sind im Praxislabor extrem variabel, am häufigsten sind eine oder zwei Analysen, aber ebenso gibt es Sitzungen, in denen mehr als 10 Parameter erhoben werden.

- Die Präsenztaxe muss zwangsläufig die für das Praxislabor nicht zutreffende Kalkulation der einzelnen Analyse kompensieren, ist aber als «pro Sitzung» definiert, ergo ein typischer Fall einer nicht stufengerechten Verwendung einer Pauschale!

\section{Point-of-Care-Ansatz als Lösung}

Mit dem POC-Ansatz schlagen die Ärzte nicht nur eine gesetzeskonforme Tarifierung vor, sondern fokussieren auch klar auf die Kernfunktion des Praxislabors - das spontan verfügbare, noch in die laufende Sitzung integrierbare Analyseresultat. Im Einzelnen:

\section{Beschränkung auf ein klares Set an Analysen} Nur Analysen, die der direkten Verwendung in der laufenden Sitzung dienen und somit das Therapieergebnis beeinflussen können, sind Teil der POC-Tarifierung. Aktuell umfasst der POC rund 30 Positionen, im Vergleich zu den weit über tausend Positionen der Analysenliste ein sehr bescheidenes Set. Die POC-Positionen sollen von allen Leistungserbringern, die ein Präsenzlabor betreiben, angewendet werden dürfen, also auch von auf Präsenzdiagnostik angewiesenen Abteilungen der Spitäler.

\section{Spezifische Kalkulation anhand der Praxislabordaten}

Zur Kalkulation der POC-Positionen werden aktuelle Daten der Praxislabors verwendet. Dazu gehören neben den spezifisch für die Präsenzdiagnostik geeigneten Kleingeräten auch die effektiv marktgängigen Preise für die Reagenzien - und zwar in Portionen fürs Praxislabor und eben nicht fürs Grosslabor - und ebenso der Aufwand der fürs Praxislabor ausgebildeten MPA, die ein Einzelresultat ermittelt.

\section{Keine Pauschalierungen und Grundtaxen}

Wie bereits in der Kritik am Vorschlag des BAG ausgeführt, sind Pauschalierungen und Grundtaxen aufgrund der Polyvalenz des Praxislabors nicht zulässig.

\section{Point-of-Care-Tarifierung}

Das Kalkulationsmodell ist ausgesprochen transparent aufgebaut und damit leicht verständlich und adaptierbar. Im Gegensatz zum geheimen Modell des BAG ist dieses Modell öffentlich, es gibt nichts zu verbergen. Als Basis dienen weitgehend die Daten aus der 2006 durchgeführten Workflowanalyse II (WFA II), die das BAG für seine Berechnung der Präsenztaxe verwendet und somit anerkennt.

\section{Relationen}

Die gesamte Kalkulation kann mit lediglich drei Relationen beschrieben werden:

\section{Positionsliste}

Liste der zu kalkulierenden Positionen mit den Variablen:

\begin{tabular}{|c|c|}
\hline Datum & Herkunft \\
\hline MPA Zeit & Mittelwerte der Messungen aus WFA-II pro Kategorie \\
\hline Kostensatz MPA & Klassische Berechnung eines Personalkostensatzes \\
\hline Kostensatz Arzt & Berechnung einer Kontrolltaxe «Laborleiter» nach TARMED-Ansatz \\
\hline Preis Reagenzien & Marktumfrage R. Fried im Verlaufe des Septembers 2008 \\
\hline Anzahl Untersuchungen & Untersuchungszahlen pro Kategorie aus WFA II \\
\hline Gerätekosten & Gerätekosten aus WFA II \\
\hline GK (Gemeinkosten) & Gemeinkostenberechnung gemäss Indirektsparte des TARMED-Kostenmodelles \\
\hline Raumkosten & Raumkosten Labor nach WFA II \\
\hline
\end{tabular}




\begin{tabular}{|c|c|}
\hline Resultatselement & Berechnung \\
\hline MPA & [Positionsliste.MPA_Zeit] $\times[$ Eckwerte.Kostensatz MPA] \\
\hline Reagenzie & [Positionsliste.Preis Reagenzie] $(\times[$ Mengenfaktor $]$ ) \\
\hline FixKosten & $\begin{array}{l}\text { [Kategorie.Gerätekosten] / [Kategorie.Anzahl Untersuchungen] + } \\
\text { [Eckwerte.Raumkosten] / } \Sigma \text { [Kategorie.Anzahl Untersuchungen] }\end{array}$ \\
\hline Arzt & [Eckwerte.Kostensatz Arzt] \\
\hline GK & [Positionsliste.MPA_Zeit] × [Eckwerte. Kostensatz GK] \\
\hline Preis & MPA + Reagenzie + FixKosten + Arzt + GK \\
\hline
\end{tabular}

\section{Abbildung 1}

Auswirkungen des Point-of-Care-Tarifes.

\begin{tabular}{|c|c|c|c|c|c|c|c|c|c|}
\hline & Pos & $\begin{array}{l}\text { Verteilung } \\
\text { nach WF- } \\
\text { Analyse }\end{array}$ & Tp alt & Tpneu & Tpwalt & TPWneu & Preis alt & Preis neu & \\
\hline Klinische Chemie & 8249.00 (z.B) & $61.00 \%$ & 9 & 9.99 & 0.90 & 1.00 & 8.10 & 9.99 & \\
\hline Gerinnung & 8548.00 & $6.00 \%$ & 14 & 10.58 & 0.90 & 1.00 & 12.60 & 10.58 & \\
\hline Hämatogr. III & 8270.00 & $11.10 \%$ & 20 & 16.61 & 0.90 & 1.00 & 18.00 & 16.61 & \\
\hline Blutsenkung & 8519.00 & $5.40 \%$ & 6 & 6 & 0.90 & 1.00 & 5.40 & 6.00 & \\
\hline CRP & 8137.00 & $7.70 \%$ & 23 & 13.78 & 0.90 & 1.00 & 20.70 & 13.78 & \\
\hline HbA1c & 8265.00 & $2.80 \%$ & 30 & 23.89 & 0.90 & 1.00 & 27.00 & 23.89 & \\
\hline \multirow{4}{*}{ Urinteilstatus } & 8580.00 & $6.00 \%$ & 4 & 4.3 & 0.90 & 1.00 & 3.60 & 4.30 & \\
\hline & & & & & & & & & \\
\hline & & & & & & & & & \\
\hline & $\begin{array}{l}\text { Preis ALT pro } \\
\text { US gewichtet }\end{array}$ & $\begin{array}{l}\text { Preis NEU pro } \\
\text { US gewichtet }\end{array}$ & Delta [Sfr] & Delta [\%] & & & & & \\
\hline Klinische Chemie & 4.94 & 6.09 & 1.15 & $23.3 \%$ & & & & & \\
\hline Gerinnung & 0.76 & 0.63 & -0.12 & $-16.0 \%$ & & & & & \\
\hline Hămatogr. III & 2.00 & 1.84 & -0.15 & $-7.7 \%$ & & & & & \\
\hline Blutsenkung & 0.29 & 0.32 & 0.03 & $11.1 \%$ & & & & & \\
\hline CRP & 1.59 & 1.06 & -0.53 & $-33.4 \%$ & & & & & \\
\hline HbA1c & 0.76 & 0.67 & -0.09 & $-11.5 \%$ & & & & & \\
\hline Urinteilstatus & 0.22 & 0.26 & 0.04 & $19.4 \%$ & & & & & \\
\hline Pro Untersuch & 10.55 & 10.88 & 0.33 & $3.1 \%$ & & & & & \\
\hline & & & & & & & & & \\
\hline
\end{tabular}

- Nettoeinkaufspreis der benötigten Reagenzien;

- Dauer der Analyse (ohne Probeentnahme).

\section{Geräteliste}

Liste der verwendeten Geräte mit den Variablen:

- Kosten pro Gerät (Unterhalt, Qualitätssicherung, Abschreibung);

- Anzahl Analysen pro Gerät.

\section{Eckwerte}

Eckwerte des Kalkulationsmodells:

- Kostensatz MPA;

- Kostensatz Arzt;

- Gemeinkostenanteil;

- Raumkostensatz.

\section{Datengrundlagen}

Sämtliche für die Kalkulation verwendete Daten sind neueren Datums und breit abgestützt erhoben (Tab. 1).

\section{Auswirkung des Point-of-Care-Tarifes}

Die Auswirkungen des POC-Tarifes lassen sich genau berechnen. Im Vergleich zum heute gültigen Tarif liegen die mengengewichteten Preise um 3,1\% höher als beim aktuellen Tarif (Abb. 1).

Die Kontrollrechnung als Vergleich zwischen dem heute angewendeten Tarif und dem neuen POC-Tarif zeigt trotz erheblicher Preisdifferenzierung in den Analysekategorien mengengewichtet kaum grosse Auswirkungen (3\%) auf die Gesamtkosten. Dieses Resultat ist glücklich, aber keineswegs konstruiert. 